\title{
UN MODELO DIFUSIÓN-ADVECCIÓN PARA LA PROPAGACIÓN DE Toxo- plasma gondii
}

\author{
A DIFFUSION-ADVECTION MODEL FOR Toxoplasma gondii PROPAGATION
}

Irene Duarte Gandica

Escuela de Investigación en Biomatemática. Universidad del Quindio, iduarte @uniquindio.edu.co

Recibido: Noviembre 1 de 2011

Aceptado: Marzo 30 de 2012

*Correspondencia del autor . Universidad del Quindío. Cra. 15 Calle 12 Norte. Armenia, Quindío, Colombia.

e-mail: iduarte@uniquindio.edu.co

\section{RESUMEN}

La toxoplasmosis es una infección causada por el parásito Toxoplasma gondii, que afecta una amplia población animal y humana; puede causar daños severos cuando es contraída congénitamente o en personas inmuno-suprimidas. Los felinos son los hospederos definitivos y animales de sangre caliente son hospederos intermediarios. Los felinos esparcen ooquistes de T. gondii después de consumir presas (roedores y aves) y agua contaminados. Los humanos se infectan con T. gondii principalmente cuando ingieren carne cruda que contiene quistes tisulares viables o cuando ingieren alimentos o agua contaminada con ooquistes de las heces de gatos infectados.

Este trabajo presenta un sistema en ecuaciones diferenciales parciales que modela la propagación del parásito a través de gatos. El modelo propuesto es una adaptación de un modelo clásico compartimental tipo SIR al que se le ha agregado dispersión de $T$. gondii y, adicionalmente, un término advectivo que representa el transporte por agua del parásito, simulando el hipotético caso de que un felino infectado deposite formas infectantes en el suelo o agua y éstas sean transportadas a través del agua, por la lluvia y/o los ríos. El sistema es resuelto numéricamente y se presentan resultados de simulaciones con varios campos de velocidades.

Palabras clave: Difusión, Advección, Toxoplasma gondii, Dispersión, Transporte por agua, Campo de velocidades.

\begin{abstract}
:
Toxoplasmosis is an infection caused by the parasite Toxoplasma gondii, affecting an extensive human and animal population. It can cause severe damages when is contracted congenitally or in immunosuppressed people. The cats are the definitive hosts and warm-blooded animals are the intermediary hosts. The cats spread $T$. gondii oocysts after consuming preys (rodents and birds) and contaminated water. Humans are infected with T. gondii, mainly when raw meat containing viable tissue cysts is consumed, or when contaminated food or water with oocysts from the feces of infected cats are consumed.

This work presents a system in partial differential equations that models the propagation of the parasite through cats. The proposed model is an adaptation of a classical compartment model type SIR to which dispersion of T. gondii has been aggregated, and additionally, an advective term that represents the parasite transportation by water, simulating the hypothetical case where an infected cat place infecting forms on the ground or water, and these are transported through the water, by the rain and/or the rivers. The system is solved numerically, and some simulation results with various fields of velocities are presented.
\end{abstract}

Keywords: Diffusion, Advection, Toxoplasma gondii, Dispersion, Transportation by water, Field of velocities. 


\section{INTRODUCCIÓN:}

La toxoplasmosis es una zoonosis parasitaria de amplia distribución mundial, que infecta una gran proporción de poblaciones humanas y animales, producida por el protozoario Toxoplasma gondii. Algunos individuos corren un alto riesgo de enfermedad grave o fatal debido a éste parásito; se incluyen los fetos y recién nacidos con infección congénita y las personas con deterioro inmunológico. La toxoplasmosis congénita es el resultado de la infección materna adquirida durante la gestación; puede provocar abortos, malformaciones congénitas, retardo mental y ceguera. De otro lado, los individuos inmunosuprimidos pueden desarrollar encefalitis plasmótica ó toxoplasmosis ocular (1).

T. gondii es un parásito coccidiano del que los felinos son los hospederos definitivos y los animales de sangre caliente son los hospederos intermediarios, por lo que en la mayor parte del mundo la presencia de gatos es de importancia fundamental para la dispersión del parásito. Los felinos esparcen ooquistes de T. gondii después de consumir presas (roedores y aves) y agua contaminados. Los felinos infectados pueden excretar millones de ooquistes por día, y esta eliminación puede mantenerse cerca de 10 días durante la primera infección, y pueden eliminar ooquistes posteriormente como consecuencia de una reactivación o de una reinfección; estos ooquistes pueden permanecer viables durante aproximadamente 18 meses en suelo húmedo. Los humanos se infectan con $T$. gondii principalmente cuando ingieren carne cruda que contiene quistes tisulares viables o cuando ingieren alimentos o agua contaminados con ooquistes de las heces de gatos infectados $(2,3)$. Hasta hace poco tiempo la toxoplasmosis no se consideró una enfermedad transmitida por el agua; sin embargo, en Santa Isabel do Ivai, Brasil, se asoció un brote de toxoplasmosis, que involucró 155 personas, con un reservorio de agua subterránea no filtrada (4); de otro lado, un importante brote de toxoplasmosis en humanos en Canadá, fue asociado a la contaminación de un reservorio de agua municipal, por felinos salvajes (5-7); además, recientemente $T$. gondii ha sido reportado en muchos mamíferos marinos $(8,9)$, lo que sugiere que el transporte por agua tiene más importancia de lo que se creía.

El ciclo vital del parásito $T$. gondii ocurre en tres etapas: Inicialmente, en el intestino delgado del gato como hospedero definitivo, ocurre la etapa sexuada que lleva a la producción de ooquistes que son expulsados y espo- rulan en el medio ambiente; posteriormente, dentro del hospedero intermediario, en el cual se lleva a cabo una fase asexuada en fase aguda con los taquizoitos y luego en el mismo hospedero intermediario; y, finalmente, la fase crónica con bradizoitos. En el gato ocurre tanto el ciclo sexuado como el asexuado (10).

El uso de métodos cuantitativos basados en modelos matemáticos para estudiar la dinámica de transmisión y control de las enfermedades infecciosas ha ganado importancia de forma notoria durante las últimas décadas entre los científicos y profesionales de la salud para idear programas efectivos de control e interpretar patrones epidemiológicos, ya que revelan relaciones que no son obvias a primera vista, hacen posible extraer propiedades y características de las relaciones entre los elementos que los conforman, permiten experimentar lo que en la realidad puede resultar costoso, peligroso y en ocasiones imposible y, además, son una herramienta útil para analizar la propagación y control de enfermedades infecciosas.

Cuando se tiene en cuenta la movilidad de las poblaciones dentro de un hábitat limitado, aparecen los modelos espaciales que, en epidemiología, se han utilizado, por ejemplo, para describir la velocidad geográfica de la Peste Bubónica que atacó la población humana en Europa durante los años 1347 a 1350; también en la transmisión de la rabia entre zorros en Inglaterra en el año 1885 (11).

En 2005, Trejos y Duarte (12) diseñaron un modelo matemático que describía la propagación del parásito T. gondii en una población hospedera de gatos, el cual combina el modelo de transmisión de una epidemia tipo SIR (13), con la difusión del parásito en una zona rectangular. Este modelo confirmó la importancia de los gatos en la transmisión de la infección por T. gondii, mostrando que un solo gato infectado puede llevar la infección a áreas aledañas, donde antes no existía. El coeficiente de difusión del parásito incluyó todos los factores que influyen en el transporte del parásito (aves, roedores, insectos, etc.), y el transporte por agua (lluvia, ríos, quebradas, etc.). El modelo que se presenta aquí evalúa la influencia del transporte por agua en la propagación de $T$. gondii, adicionando al modelo mencionado un término advectivo que representa la contribución del transporte por agua del parásito, simulando el hipotético caso de que un felino infectado deposite formas infectantes en el suelo o agua y éstas sean transportadas a través del agua, por la lluvia y/o los ríos, de forma in- 
dependiente con los demás mecanismos de transmisión.

Se incluyen aquí los resultados de la solución numérica del sistema, usando algunos campos de velocidades hipotéticos.

\section{MÉTODOS}

\section{El modelo matemático}

El modelo considera los siguientes supuestos:

1. Tasas de natalidad y mortalidad naturales para la población de felinos.

2. No se consideran tasas de migración de felinos.

3. La transmisión es indirecta14, se da por contacto adquirido (consumo de agua, presas o carnes infectadas) entre la concentración del parásito $T$. gondii y los felinos susceptibles.

4. El modelo describe la posibilidad de que un felino se infecte, como lineal a partir de un umbral mínimo, y constante, igual a 1 , cuando la población sobrepasa una cota superior.

5. El tiempo transcurrido entre el consumo del parásito y la expulsión del ooquiste al suelo no se tiene en cuenta.

6. La infección no induce muerte en los hospederos.

7. Las concentraciones del parásito y de la población del hospedero son variables en el espacio y en el tiempo.

8. No se tiene en cuenta la forma infectante del parásito.

9. Los gatos infectados, por medio de sus excrementos, contribuyen a la tasa de incremento del parásito en el medio ambiente.

10. Se considera una tasa de decaimiento natural del patógeno.

11. Se tiene en cuenta la inmunidad natural de los gatos, adquirida cuando el gato se infecta.

El modelo considera la población de gatos dividida en susceptibles $S=S(x, y, t)$, infectados $I=I(x, y, t)$ y recuperados o inmunes $R=R(x, y, t)$, y la población de parásitos $P=P(x, y, t)$, en el punto $(x, y)$, con $x, y$, medidos en kilómetros, y en el tiempo $t$, medido en días.

Los parámetros usados en el modelo son:

$\gamma$ : Tasa de natalidad de felinos.

$\mu$ : Tasa de mortalidad de felinos.

$1 / \eta$ : Período de infecciosidad.

$\beta$ : Número de parásitos que excreta un felino infeccioso al medio, por día.

$\theta$ : Decaimiento natural del parásito.

$D$ : Coeficiente de dispersión del parásito $\left(\mathrm{km}^{2} /\right.$ día$)$.
$\lambda(P)$ es la medida de la transmisión eficiente del parásito al felino susceptible, de modo que es la proporción de felinos susceptibles que se infectan al ingerir parásitos. Como no se tiene en cuenta la forma infectante del parásito (bradizoíto, ooquiste tisular, ooquiste y taquizoíto), se considera una distribución uniforme, es decir, la probabilidad de que un felino se infecte está dada por la función uniforme (12):

$\lambda(P)=\left\{\begin{array}{lll}0, & \text { si } & P<P_{\min } \\ \left(P-P_{\min }\right) /\left(P_{\max }-P_{\min }\right), & \text { si } & P_{\min } \leq P \leq P_{\max } \\ 1, & \text { si } & P>P_{\max }\end{array}\right.$ donde $P_{\min }$ es la cantidad mínima de parásitos que debe ingerir un felino susceptible para que haya infección y $P_{\max }$ es tal que, por encima de ésta cantidad, hay infección segura. Esta función es una aproximación de la logística con la ventaja algorítmica de que es lineal o constante (0 ó 1).

Así, $1 / \lambda(P)$ es el período promedio del estado susceptible del felino, $1 / \mu$ es el promedio de vida de los felinos, $1 / \beta$ es el tiempo promedio de excreción de ooquistes de un felino infectado y $1 / \theta$ es el período de vida del parásito en el suelo.

$\vec{V}$ denota un campo vectorial de velocidades, que representa el fenómeno del transporte a través del agua, de los parásitos.

Así, el modelo correspondiente a estos supuestos y variables es el siguiente:

Para

$$
\begin{aligned}
& S=S(x, y, t), I=I(x, y, t), R=R(x, y, t) \\
& \text { у } P=P(x, y, t),(x, y) \in \Omega \subset R^{2}, \Omega
\end{aligned}
$$

un dominio acotado, $t \in J=(0, T]$,

$$
\begin{aligned}
& \frac{\partial S}{\partial t}=\gamma(S+I+R)-(\lambda(P)+\mu) S, \\
& \frac{\partial I}{\partial t}=\lambda(P) S-(\mu+\eta) I, \\
& \frac{\partial R}{\partial t}=\eta I-\mu R
\end{aligned}
$$$$
\frac{\partial P}{\partial t}=\beta I-\theta P+D \Delta P-\mathrm{V} \cdot \nabla P,
$$ 
Con

$$
\begin{aligned}
& P(x, y, 0)=P_{0}, S(x, y, 0)=S_{0} \\
& I(x, y, 0)=I_{0}, R(x, y, 0)=R_{0} .
\end{aligned}
$$

La frontera es considerada con dos condiciones: Geográficamente, algún obstáculo impide el paso de parásitos en una de las partes, que llamamos $\Gamma_{1}$; en la otra parte, que llamamos $\Gamma_{2}$, el paso de parásitos es linealmente proporcional a la propia población. Así tenemos, respectivamente, condiciones de von Neumann y Robin homogéneas:

$$
\begin{aligned}
& \left.\frac{\partial P}{\partial \eta}\right|_{\Gamma_{1}}=0, \quad-\left.D \frac{\partial P}{\partial \eta}\right|_{\Gamma_{2}}=\left.\kappa P\right|_{\Gamma_{2}}, \\
& \Gamma_{1} \cup \Gamma_{2}=\partial \Omega, \quad \stackrel{\circ}{\Gamma_{1}} \cap \stackrel{\circ}{\Gamma}_{2}=\Phi,
\end{aligned}
$$

con $\kappa$ una constante de proporcionalidad, positiva.

\section{Solución numérica}

Existen resultados que garantizan la existencia y unicidad de soluciones para este tipo de sistemas (15); sin embargo, no se conocen métodos analíticos para obtener soluciones analíticas. Así, una opción viable es recurrir a métodos numéricos de aproximación que permiten la elaboración de algoritmos adecuados a simulaciones computacionales de las soluciones así obtenidas.

Para la solución numérica, se considera el dominio $\Omega$ como un rectángulo, es decir, se considera

$$
\Omega=\{(x, y) \in[0 . l] \times[0, h]\} .
$$

Se consideran $n x$ subdivisiones en la horizontal y $n y$ subdivisiones en la vertical, obteniendo un conjunto de $(n x+1) x(n y+1)$ nodos espaciales, de modo que se incluyan los nodos del borde, ya que ahí hay incógnitas; los subintervalos horizontales tienen longitud $\Delta x=V n x$ y los subintervalos verticales tienen longitud $\Delta y=h n y$. En las aproximaciones temporales, se divide el intervalo $(0, T]$ en $n t$ subintervalos, cada uno de longitud $\Delta t=T / n t$.

Para la aproximación espacial, se usa el método de diferencias finitas centradas y para la aproximación temporal, se usa el método de Crank-Nicolson (16). Estas herramientas se usan en todas las ecuaciones del sistema. Se considera inicialmente la ecuación para los parásitos, por sus características analíticas. En ésta ecuación se considera que, por fuerza del régimen estacionario de la consideración de largos períodos de tiempo, el divergente del campo es nulo, lo que es necesario, además, para el proceso que sigue.
Se aproxima $P(x, y, t)$ por $P\left(x_{k}, y_{j}, t_{n}\right)$, denotado $P_{i}^{(\mathrm{n})}$, con $i=(k-1) n n y+j$, para cada $k=1, \ldots, n n x$, y cada $j=1, \ldots$ ,nny, de modo que $i=1, \ldots, n n$.

Por el método de diferencias finitas, los valores de las derivadas son

$$
\begin{gathered}
\left.\frac{\partial P_{i}}{\partial x}\right|_{t_{n}} \approx \frac{P_{i+n n y}^{(n)}-P_{i-n n y}^{(n)}}{2 \Delta x},\left.\quad \frac{\partial P_{i}}{\partial y}\right|_{t_{n}} \approx \frac{P_{i+1}^{(n)}-P_{i-1}^{(n)}}{2 \Delta y}, \\
\left.\frac{\partial^{2} P_{i}}{\partial x^{2}}\right|_{t_{n}} \approx \frac{P_{i+n n y}^{(n)}-2 P_{i}^{(n)}+P_{i-n n y}^{(n)}}{\Delta x^{2}}, \\
\left.\frac{\partial^{2} P_{i}}{\partial y^{2}}\right|_{t_{n}} \approx \frac{P_{i+1}^{(n)}-2 P_{i}^{(n)}+P_{i-1}^{(n)}}{\Delta y^{2}} .
\end{gathered}
$$

Como hay un término advectivo, es posible que haya una oscilación en la solución numérica. Para evitarla, se utiliza un criterio que proporciona una condición sobre la discretización del dominio. Es la condición de Péclet (17):

$$
\frac{V_{x} \Delta x}{D} \leq 2, \quad \frac{V_{y} \Delta y}{D} \leq 2 .
$$

En casos que exigen aproximaciones temporales "compatibles" (del mismo orden), la opción inmediata es el método de Crank-Nicolson (16).

Este método consiste en hacer las aproximaciones, no en $t=t_{n}$ ó en $t=t_{(n+1)}$, sino en $t_{n+1 / 2}=t_{n}+\Delta t / 2$, utilizando

$$
\begin{gathered}
P\left(x_{k}, y_{j}, t_{n+\Delta t / 2}\right) \approx \frac{P_{i}^{(n+1)}+P_{i}^{(n)}}{2} \\
\frac{\partial P\left(x_{k}, y_{j}, t_{n+1 / 2}\right)}{\partial t}=\frac{P_{i}^{(n+1)}-P_{i}^{(n)}}{\Delta t},
\end{gathered}
$$

donde $i=(k-1) n n y+j$ identifica el nodo $\left(x_{k}, y_{j}\right)$.

Las dos aproximaciones tienen error de orden $\Delta t^{2}$, compatible con las aproximaciones espaciales, que también son de orden 2.

Después aplicar las anteriores aproximaciones y de reagrupar los términos para separar el correspondiente al n-ésimo paso en el tiempo, se obtiene:

$$
S_{i}^{(n+1)}\left(1-\gamma \frac{\Delta t}{2}-\mu \frac{\Delta t}{2}+\lambda\left(\frac{P_{i}^{(n+1)}+P_{i}^{(n)}}{2}\right) \frac{\Delta t}{2}\right)=
$$




$$
\begin{gathered}
S_{i}^{(n)}\left[1+\gamma \frac{\Delta t}{2}+\mu \frac{\Delta t}{2}-\lambda\left(\frac{P_{i}^{(n+1)}+P_{i}^{(n)}}{2}\right) \frac{\Delta t}{2}\right]+\gamma \frac{\Delta t}{2}\left(I_{i}^{(n+1)}+I_{i}^{(n)}+R_{i}^{(n+1)}+R_{i}^{(n)}\right), \\
I_{i}^{(n+1)}\left(1+\mu \frac{\Delta t}{2}+\eta \frac{\Delta t}{2}\right)=I_{i}^{(n)}\left(1-\mu \frac{\Delta t}{2}-\eta \frac{\Delta t}{2}\right)+\lambda\left(\frac{P_{i}^{(n+1)}+P_{i}^{(n)}}{2}\right) \frac{\Delta t}{2}\left(S_{i}^{(n+1)}+S_{i}^{(n)}\right), \\
R_{i}^{(n+1)}\left(1+\mu \frac{\Delta t}{2}\right)=R_{i}^{(n)}\left(1-\mu \frac{\Delta t}{2}\right)+\eta \frac{\Delta t}{2}\left(I_{i}^{(n+1)}+I_{i}^{(n)}\right), \\
P_{i-n n y}^{(n+1)}\left(-\frac{D \Delta t}{2 \Delta x^{2}}-\frac{V_{x_{i}} \Delta t}{4 \Delta x}\right)+P_{i-1}^{(n+1)}\left(-\frac{D \Delta t}{2 \Delta y^{2}}-\frac{V_{y_{i}} \Delta t}{4 \Delta y}\right)+P_{i}^{(n+1)}\left(1+\frac{D \Delta t}{\Delta x^{2}}+\frac{D \Delta t}{\Delta y^{2}}+\frac{\theta \Delta t}{2}\right) \\
+P_{i+1}^{(n+1)}\left(-\frac{D \Delta t}{2 \Delta y^{2}}+\frac{V_{y_{i}} \Delta t}{4 \Delta y}\right)+P_{i+n n y}^{(n+1)}\left(-\frac{D \Delta t}{2 \Delta x^{2}}+\frac{V_{x_{i}} \Delta t}{4 \Delta x}\right) \\
=P_{i-n n y}^{(n)}\left(\frac{D \Delta t}{2 \Delta x^{2}}+\frac{V_{x_{i}} \Delta t}{4 \Delta x}\right)+P_{i-1}^{(n)}\left(\frac{D \Delta t}{2 \Delta y^{2}}+\frac{V_{y_{i}} \Delta t}{4 \Delta y}\right)+P_{i}^{(n)}\left(1-\frac{D \Delta t}{\Delta x^{2}}-\frac{D \Delta t}{\Delta y^{2}}-\frac{\theta \Delta t}{2}\right)+ \\
P_{i+1}^{(n)}\left(\frac{D \Delta t}{2 \Delta y^{2}}-\frac{V_{y_{i}} \Delta t}{4 \Delta y}\right)+P_{i+n n y}^{(n)}\left(\frac{D \Delta t}{4 \Delta x^{2}}-\frac{V_{x_{i} \Delta t} \Delta \Delta x}{4 \Delta x}\right)+\frac{\beta \Delta t}{2}\left(I^{(n+1)}+I^{(n)}\right) .
\end{gathered}
$$

Dada la geometría del dominio elegido, se tiene una frontera formada por segmentos horizontales y verticales, que denotamos por $\Gamma_{A R}, \Gamma_{A B}, \Gamma_{I Z}, \Gamma_{D E}$, identificando así las fronteras de arriba, abajo, izquierda y derecha, respectivamente.

Si en cada caso, $P^{*(n)}$ denota el punto que interviene en la aproximación, pero que está por fuera de las fronteras, numéricamente se tiene,

$$
\begin{gathered}
\left.\frac{\partial P_{i}^{(n)}}{\partial y}\right|_{\Gamma_{A B}}=0=\frac{P_{i+1}^{(n)}-P^{*(n)}}{2 \Delta y}, \quad \text { ó } P^{*(n)}=P_{i+1}^{(n)}, \\
\left.\frac{\partial P_{i}^{(n)}}{\partial y}\right|_{\Gamma_{A R}}=0=\frac{P^{*(n)}-P_{i-1}^{(n)}}{2 \Delta y}, \quad \text { ó } P^{*(n)}=P_{i-1}^{(n)}, \\
\left.\frac{\partial P_{i}^{(n)}}{\partial x}\right|_{\Gamma_{I Z}}=0=\frac{P_{i+n n y^{(n)}-P^{*(n)}}^{2 \Delta x}, \quad \text { ó } \quad P^{*(n)}=P_{i+n n y}^{(n)},}{\left.\frac{\partial P_{i}^{(n)}}{\partial \eta}\right|_{\Gamma_{D E}}=\left.\frac{\partial P_{i}^{(n)}}{\partial x}\right|_{\Gamma_{D E}}=-\left(\frac{k}{D}\right) P_{i}^{(n)} \approx \frac{P^{*(n)}-P_{i-n n y}^{(n)}}{2 \Delta x}, \text { ó } P^{*(n)}=-\frac{2 k \Delta x}{D} P_{i}^{(n)}+P_{i-n n y}^{(n)} .}
\end{gathered}
$$

Después de aplicar esto a los puntos en la frontera y de acuerdo con la estrategia de aproximación, los sistemas para $S, I, R$ y $P$, respectivamente, llevan a la resolución a cada paso en el tiempo, de sistemas de la forma:

$$
\begin{gathered}
\mathbb{M}_{\mathbb{S}} \mathbb{S}^{(n+1)}=\mathbb{Q}_{\mathbb{S}} \mathbb{S}^{(n)}+\mathfrak{b}_{\mathbb{S}} \\
\mathbb{M}_{\mathbb{I}} \mathbb{I}^{(n+1)}=\mathbb{Q}_{\mathbb{I}} \mathbb{I}^{(n)}+\mathfrak{b}_{\mathbb{I}} \\
\mathbb{M}_{\mathbb{R}} \mathbb{R}^{(n+1)}=\mathbb{Q}_{\mathbb{R}} \mathbb{R}^{(n)}+\mathbb{b}_{\mathbb{R}} \\
\mathbb{M}_{\mathbb{P}} \mathbb{P}^{(n+1)}=\mathbb{Q}_{\mathbb{P}} \mathbb{P}^{(n)}+\mathbb{b}_{\mathbb{P}}
\end{gathered}
$$


donde las $b$ representan vectores de $n n$ componentes y $\mathbb{M}$ y $\mathbb{Q}$ son matrices de orden nnxnn. Estas matrices tienen una gran cantidad de elementos nulos (son llamadas, en la bibliografía clásica, sparse).

Algunos de estos vectores y matrices dependen de las variables dependientes, por ejemplo, $\mathbb{M}_{S}$ y $\mathbb{Q}_{s}$ dependen de $\mathbb{P}$, lo que le da al sistema un fuerte carácter de no linealidad. Así, se tiene entonces un sistema con el siguiente esquema:

$$
\begin{aligned}
& P^{(n+1)}=\theta_{P}\left(P^{(n)}, I^{(n)}, I^{(n+1)}\right), \\
& S^{(n+1)}=\theta_{S}\left(P^{(n)}, S^{(n)}, I^{(n)}, R^{(n)}, P^{(n+1)}, I^{(n+1)}, R^{(n+1)}\right), \\
& I^{(n+1)}=\theta_{I}\left(P^{(n)}, S^{(n)}, I^{(n)}, P^{(n+1)}, S^{(n+1)}\right), \\
& R^{(n+1)}=\theta_{R}\left(I^{(n)}, R^{(n)}, I^{(n+1)}\right),
\end{aligned}
$$

Con la condición inicial

$$
P^{(0)}=P_{0}, S^{(0)}=S_{0}, I^{(0)}=I_{0}, R^{(0)}=R_{0} .
$$

Un modo de resolver la dificultad de aproximar la solución es linealizar el sistema según Rachford (1973) (18); Douglas et al. (1979) (19).

Los procesos iterativos son obtenidos mediante el siguiente algoritmo:

1. Se obtiene $P^{(*)}$ de: $P^{(*)}=\theta_{P}\left(P^{(0)}, I^{(0)}, I^{(0)}\right)$

2. Se obtiene $S^{(*)}$ de: $S^{(*)}=\theta_{S}\left(P^{(0)}, S^{(0)}, I^{(0)}, R^{(0)}, P^{(*)}, I^{(0)}\right.$, $\left.R^{(0)}\right)$

3. Se obtiene $I^{(*)}$ de: $I^{(*)}=\theta_{I}\left(P^{(0)}, S^{(0)}, I^{(0)}, P^{(*)}, S^{(*)}\right)$

4. Finalmente se obtiene $R^{(*)}$ de: $\mathrm{R}^{(*)}=\theta_{R}\left(I^{(0)}, R^{(0)}, I^{(*)}\right)$

5. Se obtiene ahora $\mathrm{P}^{(* *)}$ de: $\mathrm{P}^{(*)}=\theta_{P}\left(P^{(0)}, I^{(0)}, I^{(*)}\right)$

6. Se obtiene $S^{(* *)}$ de: $S^{(* *)}=\theta_{S}\left(P^{(0)}, S^{(0)}, I^{(0)}, R^{(0)}, P^{(* *)}\right.$, $\left.I^{(*)}, R^{(*)}\right)$

7. Se obtiene $I^{(* *)}$ de: $I^{(* *)}=\theta_{I}\left(P^{(0)}, S^{(0)}, I^{(0)}, P^{(* *)}, S^{(* *)}\right)$

8. Finalmente se obtiene $R^{(* *)}$ de: $R^{(* *)}=\theta_{R}\left(I^{(0)}, R^{(0)}\right.$, $\left.I^{(* *)}\right)$

9. Procediendo de forma análoga, se obtienen sucesivamente $P^{(* * *)}, S^{(* * *)}, I^{(* * *)}$ y $R^{(* * *)}$, hasta que se definen las aproximaciones de los vectores $P^{(1)}, S^{(1)}, I^{(1)}$ y $R^{(1)}$.

10. Los pasos 1. a 9. Se repiten con $P^{(\mathrm{n})}, S^{(\mathrm{n})}, I^{(\mathrm{n})}$ y $R^{(\mathrm{n})}$, en lugar de $P^{(0)}, S^{(0)}, I^{(0)}$ y $R^{(0)}$, respectivamente, para obtenerse, después de las sucesivas iteraciones internas, el $(n+1)$-ésimo paso en la iteración temporal, $P^{(n+1)}, S^{(n+1)}, I^{(n+1)}$ y $R^{(\mathrm{n}+1)}$.
Generalmente no hay ganancias cuando se repiten muchas veces estas iteraciones internas en cada paso del tiempo, siendo suficientes 4 veces $(18,19)$.

Este método de tipo predictor-corrector, definido en el ámbito de una discretización Crank-Nicolson, mejora las aproximaciones, pero no indefinidamente: él tiende a la mejor aproximación de orden $(\Delta t)^{2}$ en cada iteración temporal (resultados de convergencia se pueden encontrar en los trabajos citados) $(18,19)$.

Este esquema de orden cuadrático desde el punto de vista espacial global, es de orden también cuadrático temporalmente, pero desde el punto de vista local.

\section{RESULTADOS Y DISCUSIÓN}

El algoritmo fue programado en ambiente Matlab.

Los valores de los parámetros usados en las simulaciones son:

- $\quad$ Tasa de natalidad de felinos $\gamma=0,024$

- Tasa de mortalidad de felinos $\mu=0,02$

- Tasa de recuperación de gatos infectados $\eta=0,6$

- Número de parásitos que excreta un felino infeccioso al medio, por día, se calculó teniendo en cuenta el número de ooquistes que expulsa un gato infecciosos durante su período de infecciosidad, $\beta=10$ (en unidades de millón)

- Decaimiento natural del parásito $\theta=0,05$

- Coeficiente de dispersión del parásito $\left(\mathrm{km}^{2} /\right.$ día $)$, $D=5 \times 10^{-2} x 0,0864$.

La cantidad de parásitos mínima que debe consumir un gato susceptible para infectarse es de $P_{\min }=10,000$ y por encima de $P_{\max }=1,000,000$ hay infección segura.

Las simulaciones están hechas en una malla de $n x=41 \mathrm{y}$ $n y=57$. Inicialmente, en cada nodo de la malla, hay un gato infectado, 10 gatos susceptibles, cero recuperados y cero parásitos.

Las figuras 1 a 4 corresponden al comportamiento de las poblaciones con 4 campos de velocidades hipotéticos diferentes. Las figuras a presentan el campo de velocidades, indicando el lugar donde se coloca el gato infectado inicial (GI) y otro punto de referencia (S); éste punto de referencia permite hacer una comparación de la evolución de la infección en puntos "cerca" y "lejos" del inóculo; es escogido de modo que el parásito, por efecto de la difusión y el campo de velocidades del agua, alcance a llegar hasta allí, en las 100 iteraciones. Las figuras b representan el comportamiento de las po- 
blaciones después de 100 iteraciones, correspondiente al campo de las figuras a. Las figuras c presentan el comportamiento a través del tiempo, de las poblaciones de susceptibles e infectados en los puntos donde está el gato infectado inicial y en el punto de referencia.

En los 4 casos se observa claramente la propagación de las poblaciones en la dirección del campo de velocidades. Los parásitos se diseminan como consecuencia de la difusión y, adicionalmente, del transporte a través del agua.

El campo de velocidades mostrado en la figura 1.a tiene velocidad positiva en $x, 0.3365 \mathrm{~km} /$ día, y cero en $y$. Correspondientemente, las poblaciones aumentan en ésta dirección (figura 1.b). Se observa que en el lado izquierdo del lugar donde se coloca el gato infectado no hay cambios en las poblaciones, exceptuando los gatos susceptibles, cuya población disminuye leve y uniformemente, debido a que la tasa de mortalidad supera la de natalidad. La figura 1.c muestra cómo la población de infectados es mayor en el lugar del inóculo (GI), que en el punto de referencia (S); esto, debido al tiempo que tardan las corrientes de agua en llevar los parásitos a tal punto. Correspondientemente, la población de susceptibles disminuye más lentamente en el lugar del inóculo que en el punto de referencia.

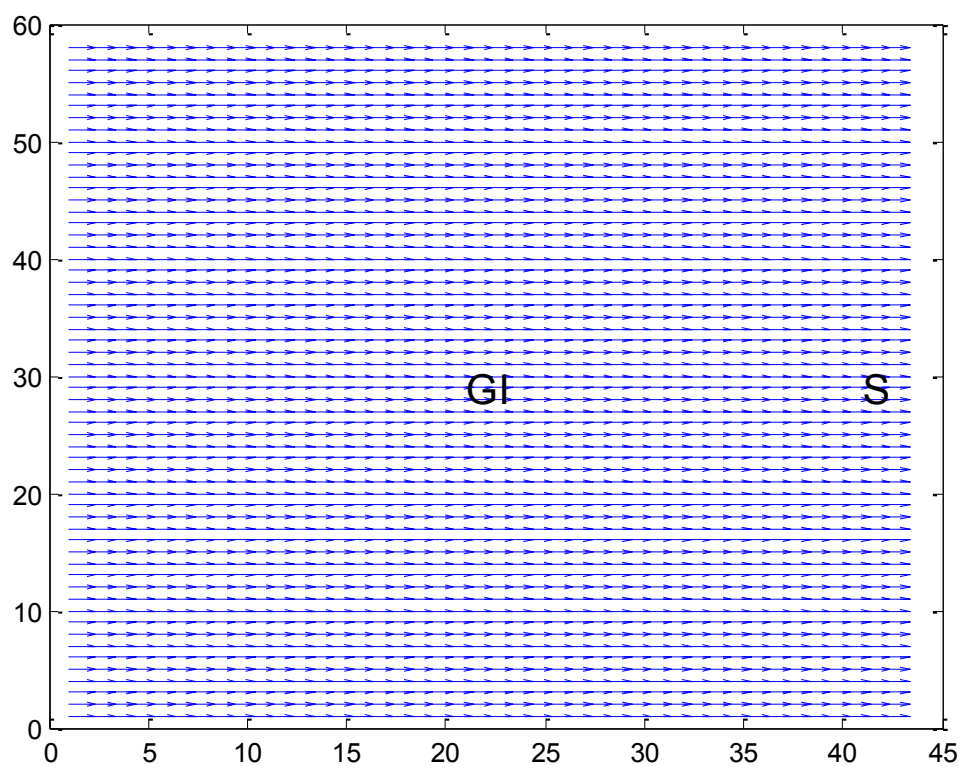

Figura 1. a. Campo de velocidades con dirección positiva en $x$ y cero en y. GI indica el lugar donde se coloca el único felino infectado inicialmente y $\mathrm{S}$ es un punto de referencia. 
Gatos Susceptibles

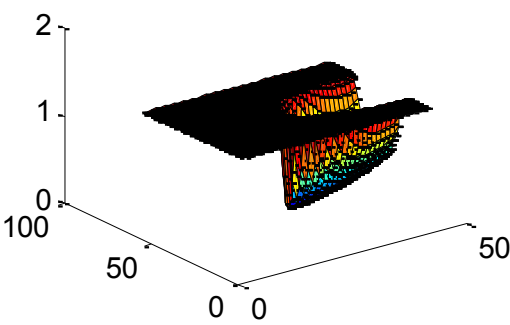

Gatos Recuperados

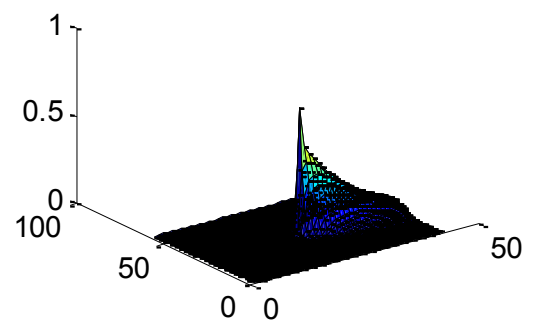

Gatos Infectados

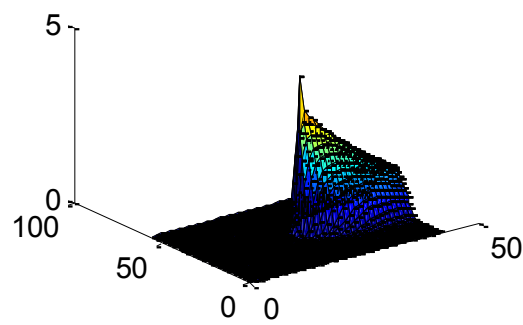

Parásitos (en millones)

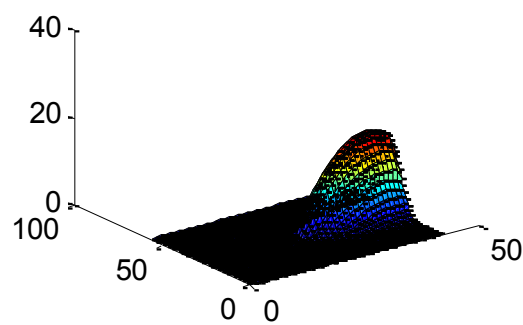

Figura 1. b. Comportamiento de las poblaciones, correspondiente al campo de velocidades de la figura 1.a, después de 100 iteraciones.
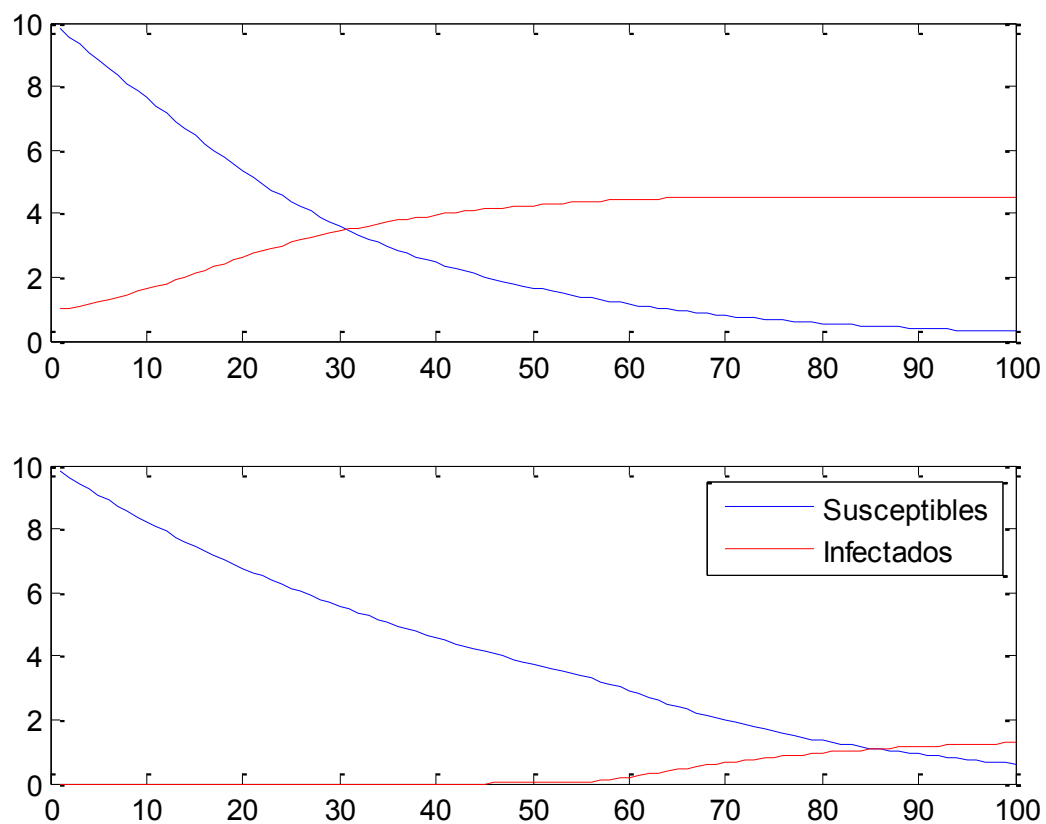

Figura 1. c. Comportamiento de las poblaciones de felinos susceptibles e infectados a través del tiempo, cuando el campo de velocidades es del tipo de la figura 1.a: En el lugar del inóculo GI (gráfica superior) y en un lugar $\mathrm{S}$ en el medio de la frontera derecha 
El campo de velocidades mostrado en la figura 2.a tiene velocidad cero en $x$ y positiva en $y, 0.3222 \mathrm{~km} /$ día. Correspondientemente, la figura 2.b muestra el desplazamiento de las poblaciones en ésta dirección, observándose un comportamiento similar al del caso anterior. Nótese en la figura 2.c, que la población de infectados en el punto de referencia comienza a crecer a los 80 días aproximadamente, mientras que en el caso anterior comenzaba a crecer antes de los 60 días; esto, debido a que la velocidad en éste caso es mayor.

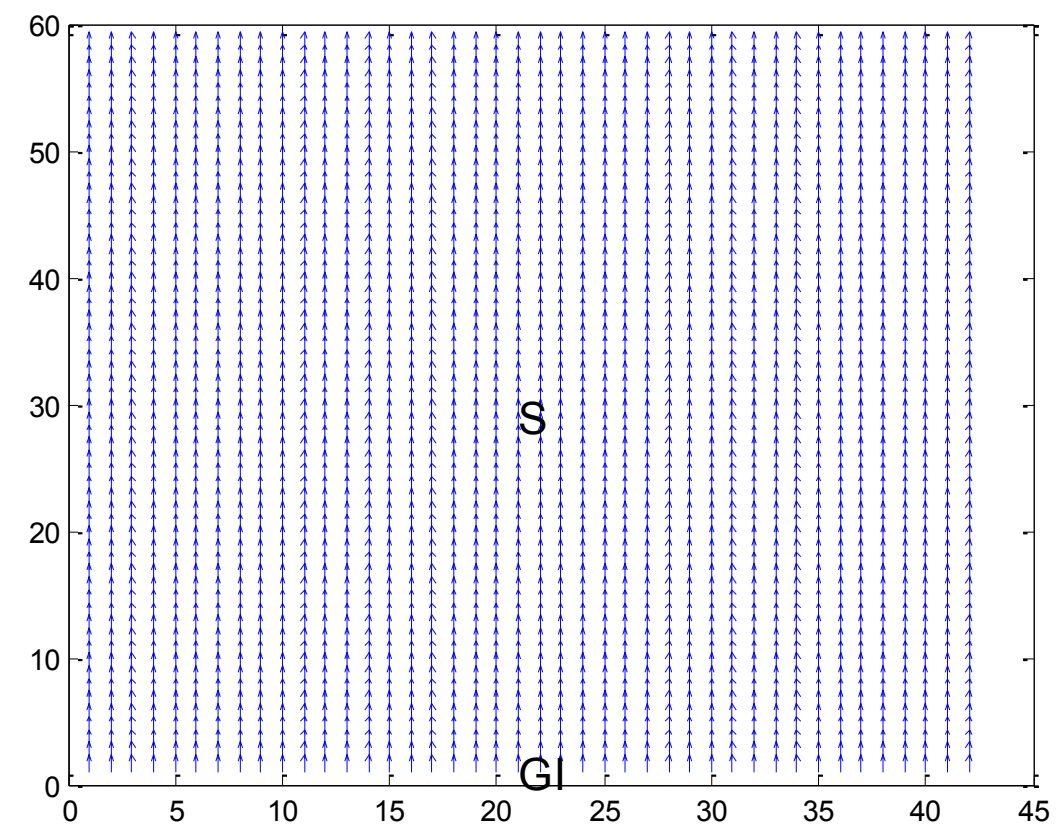

Figura 2. a. Campo de velocidades con velocidad cero en $\mathrm{x}$, positiva en y. GI indica el lugar donde se coloca el único felino infectado inicialmente, y $\mathrm{S}$ es un punto de referencia.

\section{Gatos Susceptibles}

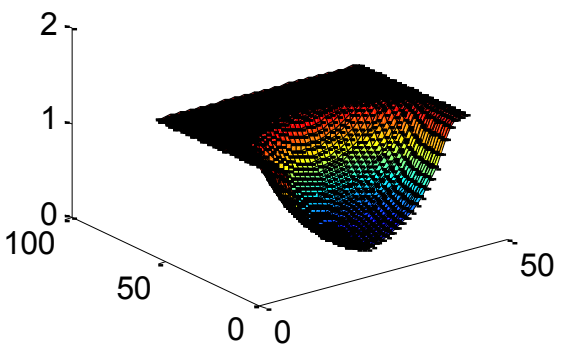

Gatos Recuperados

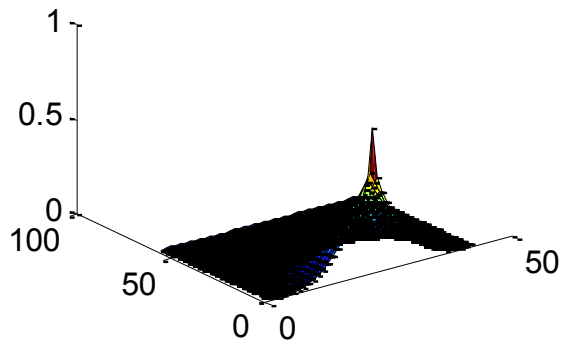

\section{Gatos Infectados}

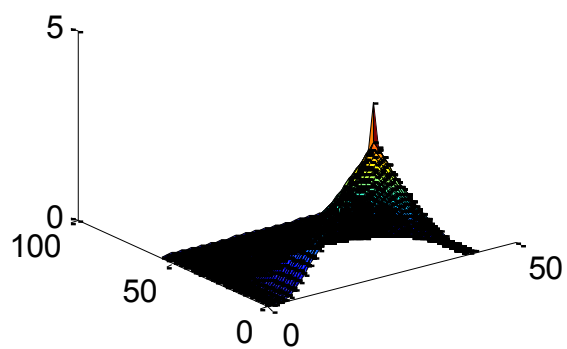

Parásitos (en millones)

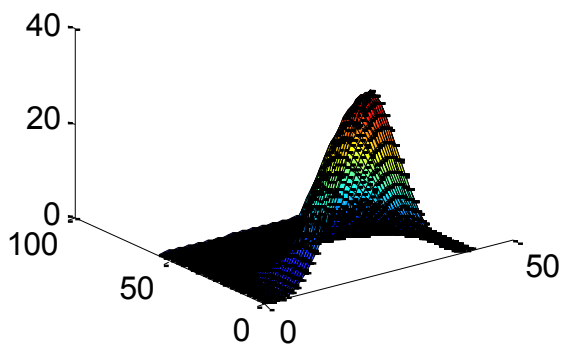

Figura 2. b. Comportamiento de las poblaciones, correspondiente al campo de velocidades de la figura 2.a, después de 100 iteraciones. 

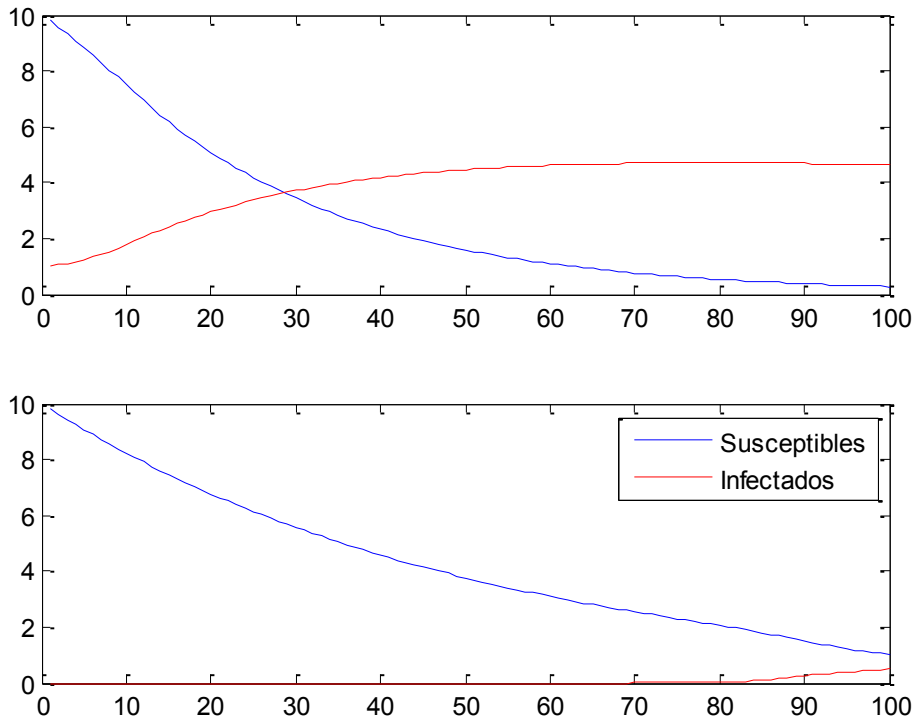

Figura 2. c. Comportamiento de las poblaciones de felinos susceptibles e infectados a través del tiempo, cuando el campo de velocidades es del tipo de la figura 2.a., en el lugar del inóculo GI (gráfica superior) y en el lugar $\mathrm{S}$ en el centro de la región (gráfica inferior).

El campo de velocidades mostrado en la figura 3.a tiene velocidades negativa en $x, 0.3365 \mathrm{~km} /$ día y negativa en $y$, $0.3222 \mathrm{~km} /$ día. Corrspondientemente, las poblaciones varían en esa dirección, hacia el origen (figura 3.b).

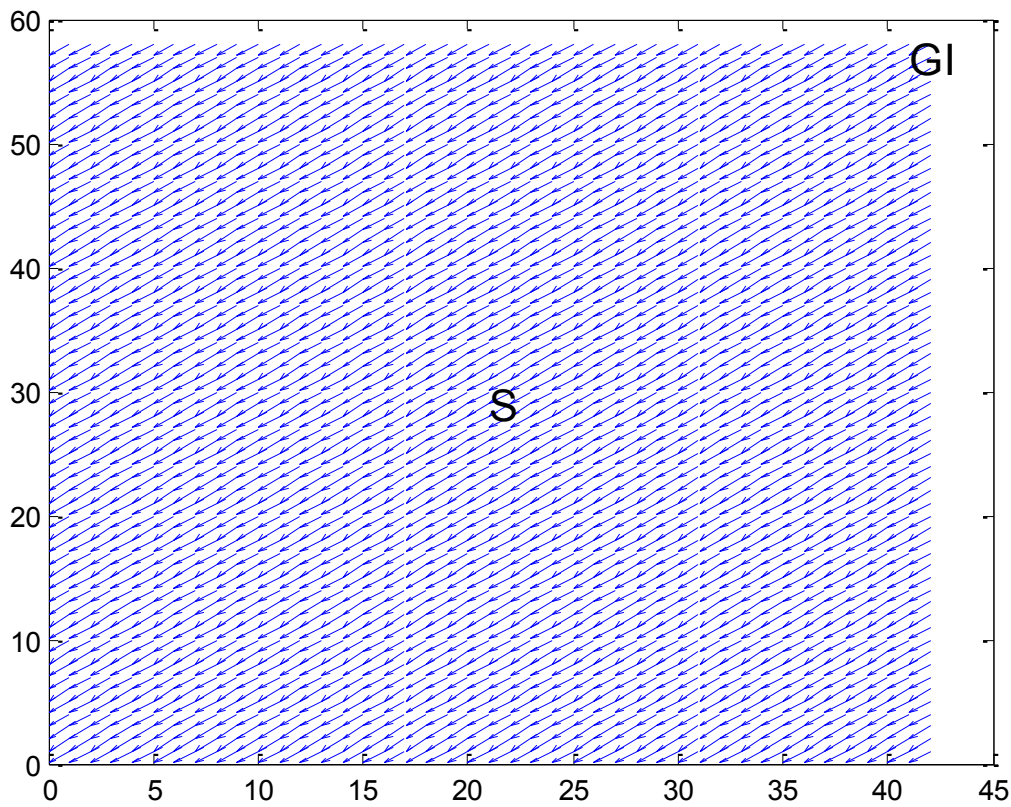

Figura 3. a. Campo de velocidades con velocidad negativa en $\mathrm{x} y$ negativa en y. GI indica el lugar donde se colocó el único felino infectado inicialmente y $\mathrm{S}$ es un punto de referencia. 
Gatos Susceptibles

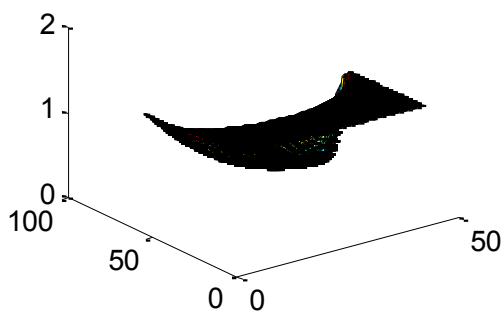

Gatos Recuperados

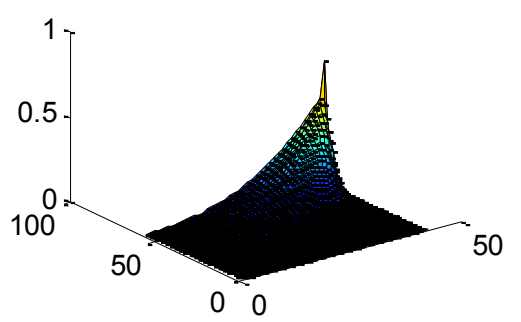

Gatos Infectados

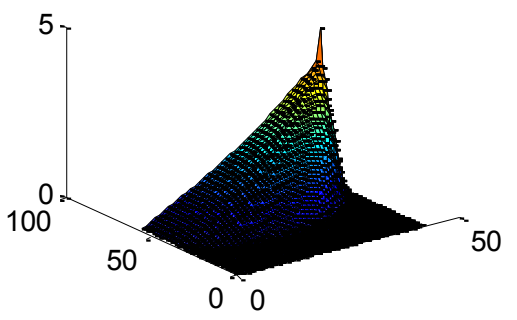

Parásitos (en millones)

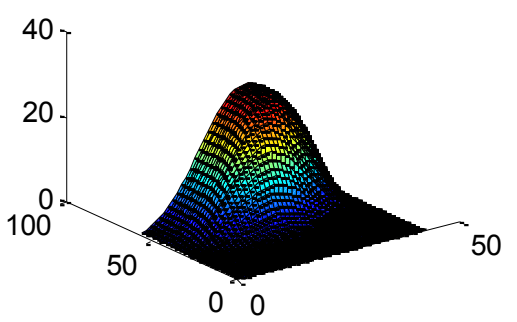

Figura 3. b. Comportamiento de las poblaciones, correspondiente al campo de velocidades de la figura 3.a, después de 100 iteraciones.
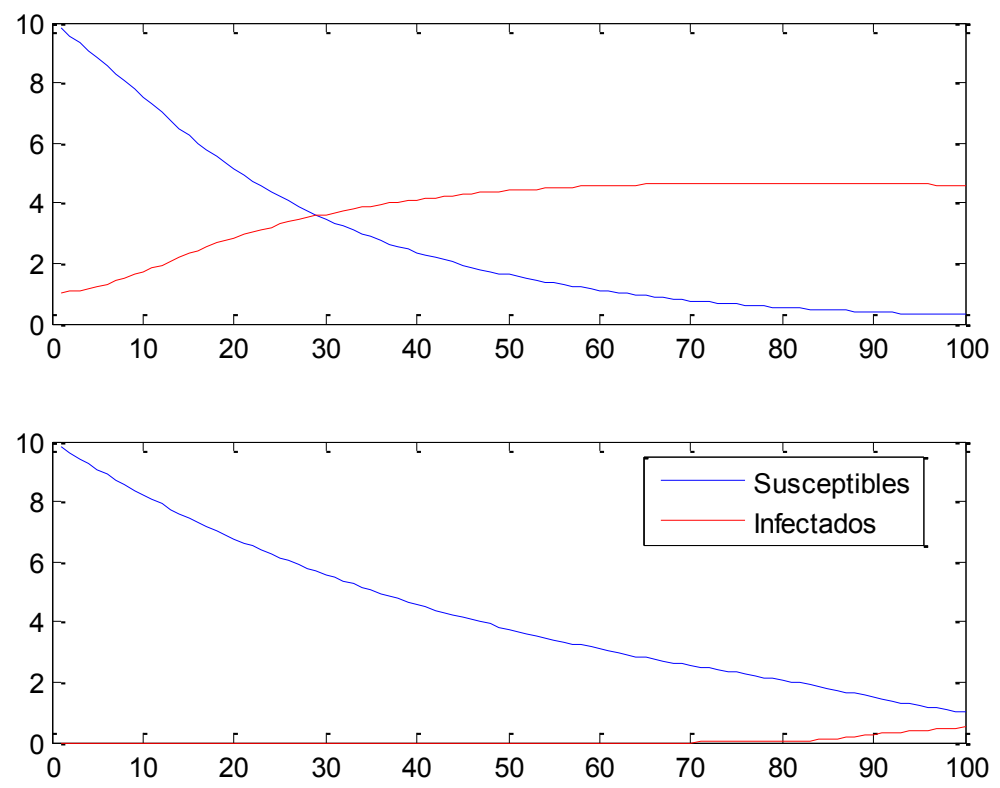

Figura 3. c. Comportamiento de las poblaciones de felinos susceptibles e infectados a través del tiempo, cuando el campo de velocidades es del tipo de la figura 3.a., en el lugar del inóculo GI (gráfica superior) y en un lugar $\mathrm{S}$ en el centro de la región (gráfica inferior). 
El campo de velocidades mostrado en la figura 4.a tiene velocidades diferentes en 4 subregiones. Consecuentemente, las poblaciones se mueven en las direcciones correspondientes y se observa el cambio cuando las poblaciones pasan de una subregión a otra, (figura 4.b).

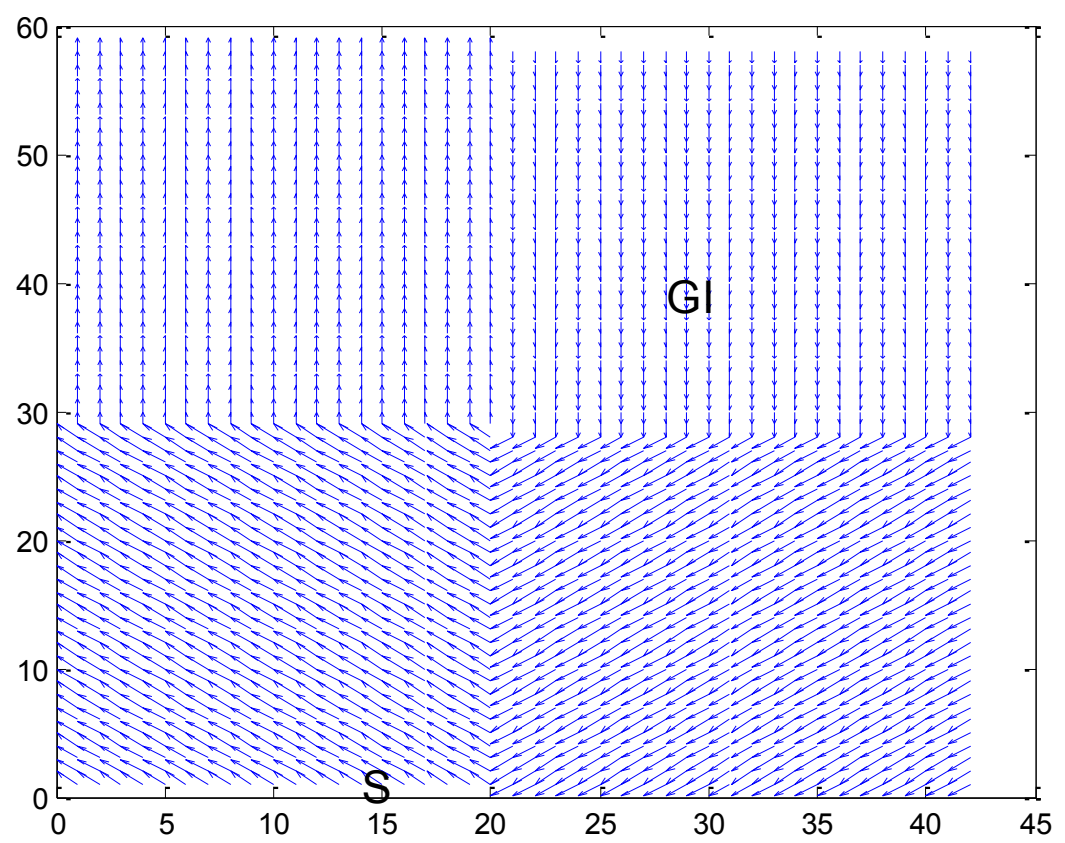

Figura 4. a. Campo de velocidades con velocidades diferentes en 4 subregiones. GI indica el lugar donde se coloca el único felino infectado inicialmente y $\mathrm{S}$ es un punto de referencia.

Gatos Susceptibles

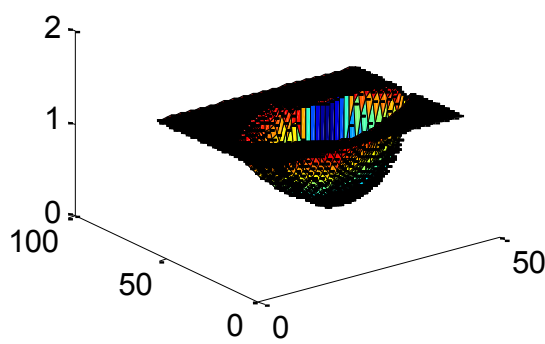

Gatos Recuperados

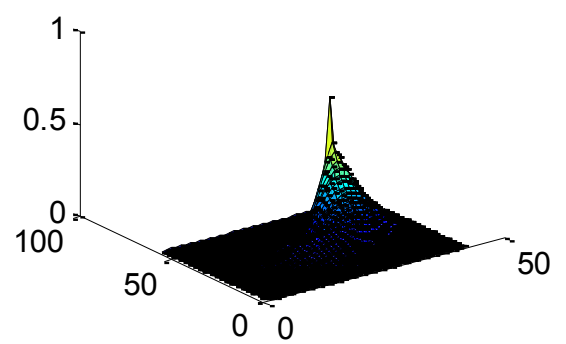

Gatos Infectados

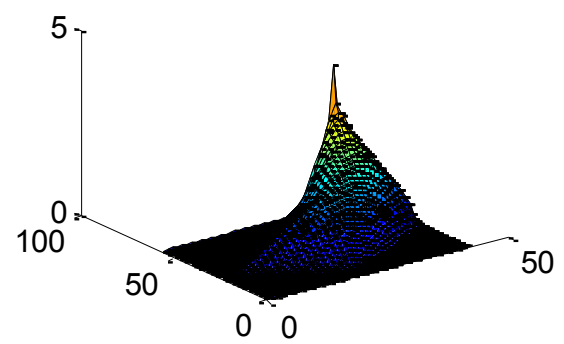

Parásitos (en millones)

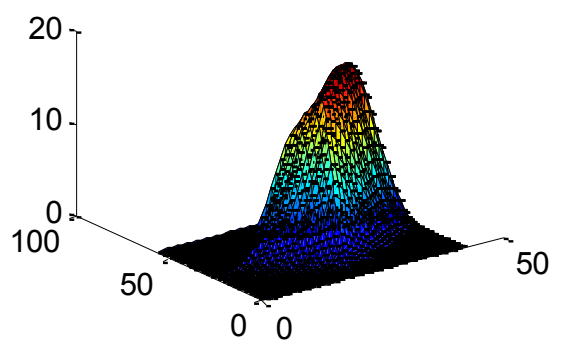

Figura 4. b. Comportamiento de las poblaciones, correspondiente al campo de velocidades de la figura 4.a, después de 100 iteraciones. 

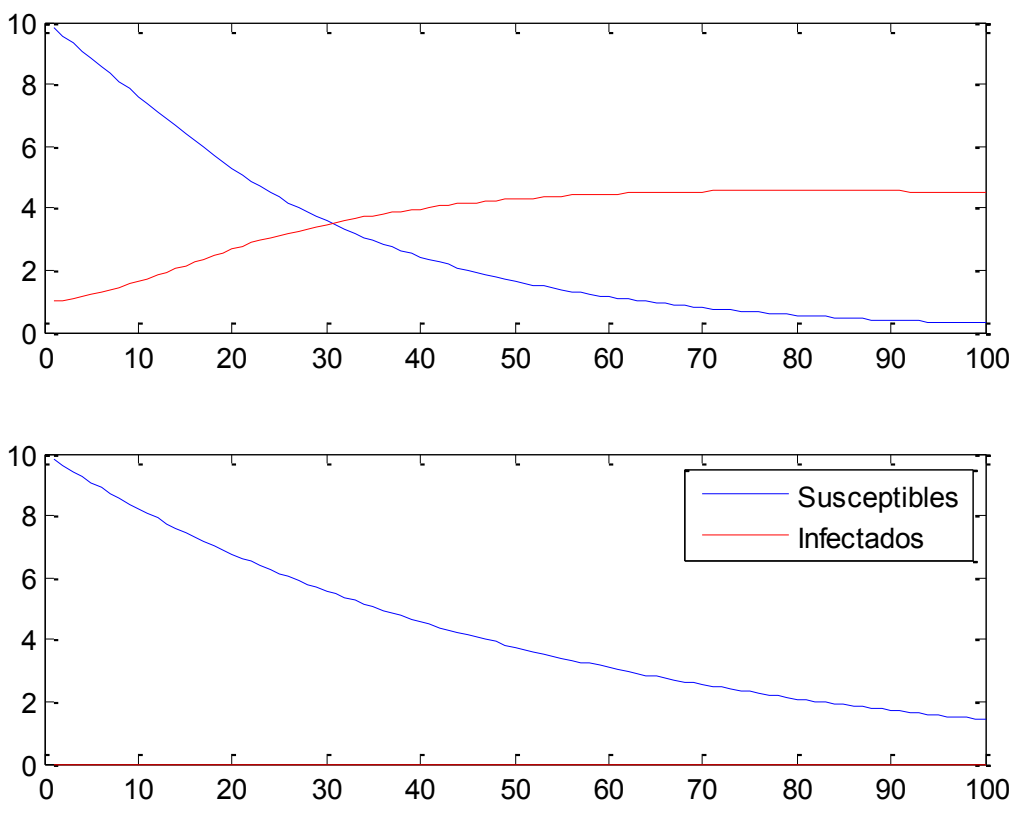

Figura 4. c. Comportamiento de las poblaciones de felinos susceptibles e infectados a través del tiempo, cuando el campo es del tipo de la figura 4.a., en el lugar del inóculo GI (gráfica superior) y en un lugar S en la frontera inferior de la región (gráfica inferior).

\section{CONCLUSIONES}

A pesar de las simplificaciones hechas, el modelo muestra buenos resultados en términos de la descripción de la dinámica de la propagación de $T$. gondii y mejora, al incluir el término advectivo que representa el transporte por agua del parásito, el modelo propuesto originalmente por Trejos y Duarte (12).

Este trabajo permite corroborar el hecho de que un solo gato infectado puede propagar el parásito T. gondii a áreas aledañas donde no estaba presente, y el hecho de que el agua contaminada tiene el potencial de dispersar la infección a un gran número de personas. El modelo, además, permite prever los lugares hacia los que el parásito se moverá por acción del transporte por agua (lluvia o ríos), en el caso en que un felino infecte los suelos, presas o agua potable, a través de sus heces. Si se tiene un campo de velocidades real de alguna región en estudio, se puede incorporar al algoritmo numérico sin dificultad.

En términos de la infección, se concluye que para ayudar a prevenir la contaminación del ambiente con ooquistes, se debe pensar en métodos para filtrar $T$. gondii en las plantas de tratamiento de aguas y en métodos para inactivar el parásito depositado en el suelo a través de las heces del gato, de modo que no se disperse en el ambiente. 


\section{BIBLIOGRAFIA}

1. Mandell, G.L., Bennett, J.E., y Dolin, R. Principios y prácticas en enfermedades infecciosas. Vol. 2. Editorial Médica Panamericana. $5^{\mathrm{a}}$ ed. 2000.

2. Frenkel, J.K., y Dubey, J.P. "Toxoplasmosis and its prevention in cats and man". En Journal of Infectious Diseases, 1972; 126,664-673.

3. Jones, J.L., y Dubey J.P. (). "Waterborne toxoplasmosis-Recent developments". En Experimental Parasitology, 2010; 124,10-25.

4. de Moura, L., Bahia-Oliveira, L.M.G., Wada, M.Y., Jones, J.L., Tuboi, S.H., Carmo, E.H., Ramalho, W.M., Camargo, N.J., Trevisan, R., Graça, R.M.T., da Silva, A.J., Moura, I., Dubey, J.P., y Garrett, D,O. "Waterborne outbreak of toxoplasmosis, Brazil, from field to gene". En Emerging Infectious Diseases. 2006; 12,326-329.

5. Bowie, W.R., King, A.S., Werker, D.H., Isaac-Renton, J.L., Bell, A., Eng, S.B., y Marion, S.A. “Outbreak of toxoplasmosis associated with municipal drinking water". En Lancet, 1997;350,173-177.

6. Isaac-Renton, J., Bowie, W.R., King, A., Irwin, G.S., Ong, C.S., Fung, C.P., Shokeir, M.O., y Dubey, J.P. "Detection of Toxoplasma gondii oocysts in drinking water". En Applied and Environmental Microbiology, 1998; 64:2278-2280.

7. $\quad$ Eng, S.B., Werker, D.H., King, A.S., Marion, S.A., Bell, A., Issac-Renton, J.L., Irwin, G.S., y Bowie, W.R. "Computer-generated dot maps as an epidemiologic tool: investigating an outbreak of toxoplasmosis". En Emerging Infectious Diseases. 1999; 5:815-819.

8. Conrad, P.A., Miller, M.A., Kreuder, C., James, E.R., Mazet, J., Dabritz, H., Jessup, D.A., Gulland, F., y Grigg, M.E. "Transmission of Toxoplasma: clues from the study of sea otters as sentinels of Toxoplasma gondii flow into the marine environment". En International Journal for Parasitology, 2005; 35:1155-1168.

9. Dubey, J.P., y Jones, J.L. "Toxoplasma gondii infection in humans and animals in the United States". En International Journal for Parasitology, 2008; 38:1257-1278.

10. Gómez-Marín, J.E. Protozoología médica. Editorial El Manual Moderno. Colombia. 2010.

11. Murray, J.D. Mathematical Biology II: Spatial Models and Biomedical Applications. Springer-Verlag. New York. 2003.

12. Trejos, A.D.Y., y Duarte, G.I. "Un modelo matemático de la propagación de Toxoplasma gondii a través de gatos". En Actualidades Biológicas, 2005; 27(83):143-149.

13. Hethcote, H.W. “The Mathematics of Infectious Diseases”. En Siam review, 2000; 42(4):599-653.

14. Anderson, R., y May, R.M. Infectious diseases of humans. Oxford University Press. 1991.

15. Cantrell, R.S., y Cosner C. Spatial ecology via reaction-diffusion equations. John Wiley and Sons. Chichester, UK. 2003.

16. Burden, R.L., y Faires, J.D. Numerical Analysis. Thomson Brooks/Cole. USA. 2005.

17. Strikwerda, J.C. Finite Difference Schemes and Partial Differential Equations. Wadsworth and Brooks Cole. 1989.

18. Rachford, H.H. Two-level discrete-time Galerkin approximations for second order nonlinear parabolic partial differential equations. SIAM Journal on Numerical Analysis, 1973; 10(6):1010-1026.

19. Douglas Jr., J., Dupont, T., Ewing, R.E. Incomplete iteration for time-stepping a galerkin method for a quasi-linear parabolic problem. SIAM Journal Numerical Analysis, 1979; 16:503-522. 